\title{
Detection for All Zero Coefficient Blocks in HEVC Based on Uniform Quantizer
}

\author{
Nana SHAN ${ }^{\mathrm{a}, 1}$, Henglu WEI ${ }^{\mathrm{b}}$, Wei ZHOU ${ }^{\mathrm{c}}$, and Zhemin DUAN ${ }^{\mathrm{c}}$ \\ ${ }^{a}$ Taishan University, China \\ ${ }^{\mathrm{b}}$ Tsinghua University, China \\ ${ }^{\mathrm{c}}$ Northwestern Polytechnical University, China
}

\begin{abstract}
Transform and quantization are adopted in HEVC. There are lots of all zero coefficient blocks in transform and quantization. By detecting all zero coefficient blocks, the complexity of transform or quantization can be greatly reduced. All zero coefficient blocks for uniform quantizer can be efficiently detected by comparing the float quantization level of the estimated coefficients with an explicit threshold. The experimental result shows that $50 \%$ complexity of transform or quantization for uniform quantizer can be reduced with negligible loss of video coding efficiency.
\end{abstract}

Keywords. HEVC, all zero coefficient blocks, uniform quantization, RDO quantization, SATD

\section{Introduction}

High Efficiency Video Coding (HEVC) is the new video coding standard developed by JCT-VC [1]. HEVC provides better compression performance than H.264/AVC [2]. Several new techniques are included in HEVC, such as Rate Distortion Optimized Quantization (RDOQ), Sample Adaptive Offset (SAO), Coding Tree Unites (CTU).

Transform and quantization are the most computational parts in HEVC [3]. The basic unit for transform and quantization in HEVC is called transform block (TB). Skipping all zero coefficient blocks can reduce the coding complexity of transform and quantization, as well as the prediction mode decision and advanced motion vector prediction [4].

There are many proposed detection algorithms for all zero coefficient blocks in H.264/AVC. An early predicting all zero coefficients is proposed in [5], and the threshold can be changed with the quantization level. A hybrid model was proposed in [6] to predict zero quantized Discrete Cosine Transform (DCT) coefficients with Gaussian distribution and optimized efficient condition to detect all zero DCT blocks. To get more frequency characteristics, Xie presents a mathematic model by analysing DCT for detecting all zero coefficient blocks [7].

The above algorithms mainly focused on detecting all zero coefficient blocks by mathematic models without considering uniform quantizer (UQ). In this paper, the

\footnotetext{
${ }^{1}$ Corresponding Author, Nana Shan; School of Information Science and Technology, Taishan University. E-mail: helensnn@hotmail.com. This work was supported by the Scientific Research Foundation of Taishan University (Grant No. Y-01-2017003).
} 
detecting all zero coefficient blocks algorithm for UQ is proposed. And the proposed algorithm is based on determining the quantization level (QL) for the global maximum magnitude (MM). The estimated global MM is used to detect all zero coefficient blocks (AZCB) and non-all zero coefficient blocks (non-AZCB). As a result, AZCB can be easily detected by comparing the float QL (FQL) of the estimated coefficients with the threshold.

\section{Transform and Quantization in HEVC}

The HEVC standard specifies integer DCT matrices of size $4 \times 4,8 \times 8,16 \times 16$ and $32 \times 32$ to be used for two-dimensional transforms in the context of block-based motioncompensated video compression. For the $4 \times 4$ transform of luma intra picture prediction residuals, an integer discrete sine transform (DST) is alternatively specified. In addition, transform skip is also introduced in HEVC to improve compression ratio of screencontent video sequences generated in applications such as remote desktop, slideshows etc. When transform skip mode is used, prediction residuals are quantized directly. Transform skip mode is restricted to only $4 \times 4$ transform blocks. DST is regarded as a special DCT, and transform skip is regarded as a special transform.

By supposing the transform of HEVC encoder is orthonormal in this paper, UQ can be calculated as,

$$
l_{i}^{u q}=\text { floor }\left(\frac{\left|c_{i}\right|}{Q_{\text {step }}}+\text { offset }\right)
$$

where $\mathrm{c}_{\mathrm{i}}$ is transform coefficient, $\mathrm{l}_{\mathrm{i}}^{\mathrm{uq}}$ is the $\mathrm{QL}$ of $\mathrm{c}_{\mathrm{i}}$, and $\mathrm{Q}_{\text {step }}$ is the step of quantization. The offset depends on the slice type, for I slices the offset equals $1 / 3$ and for non-I slices it is $1 / 6$.

\section{DCT Coefficients Estimation}

Zero mean Gaussian distribution model is used in this paper to get the distribution of prediction residuals. Then, the distribution of DCT coefficients can be calculated. The standard deviation of DCT coefficients are used to estimate the global MM.

DCT coefficients are estimated twice in the original method. The proposed method use transform coefficients estimation based on the feature of DCT energy concentration. As Figure 1 shows, in the DCT process, prediction residuals are concentrated with more low frequency coefficients, low frequency coefficients have higher impacts to the coding units than high frequency coefficients. So, we should focus on high frequency coefficients.

There is a threshold of high frequency coefficients and low frequency coefficients. From the classification result shows in Figure 1, coefficients in $4 \times 4 \mathrm{~TB}$ are all selected as low frequency coefficients. But for $8 \times 8,16 \times 16$ and $32 \times 32 \mathrm{TBs}$, only part coefficients are selected as low frequency coefficients and most of them are gathered at the left-top of the coding units. 


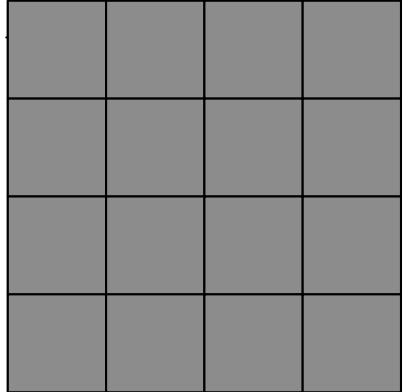

(a) $4 \times 4 \mathrm{~TB}$

10

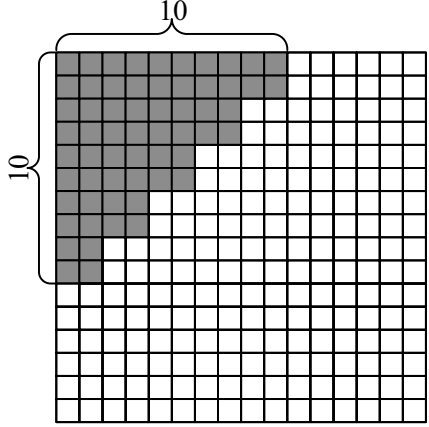

(c) $16 \times 16 \mathrm{~TB}$

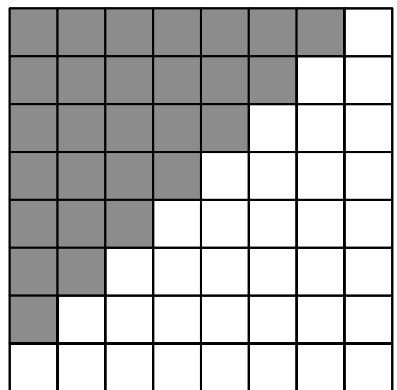

(b) $8 \times 8 \mathrm{~TB}$

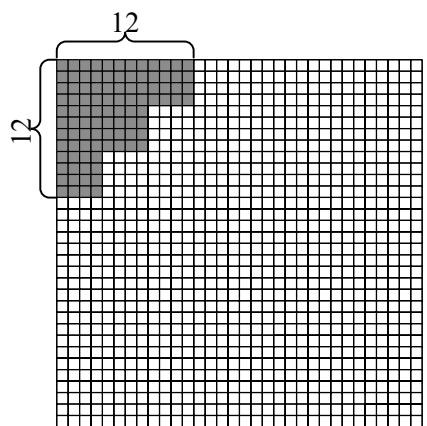

(d) $32 \times 32 \mathrm{~TB}$

Figure 1. The selected low frequency coefficients

\section{AZCB detection in HEVC}

The limit between QL equals zero and non-zero is explicit for UQ. And FQL can be defined as,

$$
l_{i}^{\text {float }}=\frac{\left|c_{i}\right|}{Q_{\text {step }}}
$$

Comparing (1) and (2), it can be found that $l_{i}^{\text {float }}$ is just the float style of $l_{i}^{u q}$. What is more, if $l_{i}^{\text {float }}$ is less than $(1-$ offset $), l_{i}^{u q}$ equals to zero. Set $T h \_U=(1-$ offset $)$, then $T h \_U$ can be used as the threshold between QL equals zero and non-zero. If $T h \_U$ is larger than FQL of every coefficient in a TB, AZCB can be determined. Therefore, the FQL of the estimated low frequency coefficients and $H M_{\text {est }}$ should be compared with $T h \_U$ one by one until AZCB or non-AZCB can be determined. But it is found that the accuracy of AZCB detection is sensitive to the MM of the estimated low frequency coefficients. The estimated low frequency coefficients (HT coefficients) are amplified a bit before AZCB detection to prevent the misclassification of the non-AZCB.

The scaling factor is calculated as,

$$
\alpha_{N}=\operatorname{mean}_{u, v \in L F}\left(\sigma_{D / H}(u, v)\right), \sigma_{D / H}(u, v)>1
$$

where $L F$ is the location set of the selected low frequency coefficients, $N$ is the size of TB and $\sigma_{D / H}=\sigma_{D} / \sigma_{H} \cdot \sigma_{D}$ and $\sigma_{H}$ are the standard deviation of DCT coefficients and the standard deviation of HT coefficients respectively. These two parameters can be 
obtained by replacing $D$ by DCT or HT. Then, the estimated low frequency DCT coefficient is,

$$
L_{e s t, N}(u, v)=\alpha_{N} \cdot B_{N}(u, v), \quad u, v \in L F
$$

Besides, the global MM can also be used to pre-detect AZCB. It is obvious that if the $\mathrm{QL}$ of the global MM is zero, the QLs of other coefficients must be zero. Therefore, AZCB can be easily determined by comparing the FQL of the global MM with $T h \_U$. If the FQL of the global MM is less than Th_U, AZCB can be determined. The estimated global MM is also used to skip coefficients estimation when non-AZCB occurs. The threshold in this paper is assigned to be 1.3 for SAD and 1.9 for SATD. If the FQL of the estimated global MM is larger than the defined threshold, denoted by Th_skip, the TB is considered as a non-AZCB, and coefficients estimation will be skipped.

The flowchart of AZCB detection in UQ is shown in Figure 2. At first, the global MM of DCT coefficients is estimated and its FQL is compared with $T h \_U$. If $T h \_U$ is larger than the FQL, AZCB is determined; if not the FQL will be compared with Th_skip to determine non-AZCB. If $\mathrm{AZCB}$ or non-AZCB cannot be determined, then low frequency coefficient $L_{e s t, N}(u, v)$ is estimated and the corresponding FQL is compared with $T h \_U$ one by one to determine non-AZCB. If non-AZCB cannot be determined by low frequency coefficients, the MM of high frequency coefficients $H M_{e s t}$ will be calculated and its FQL is compared with $T h \_U$. If $T h \_U$ is larger than the FQL, the corresponding $\mathrm{TB}$ is an $\mathrm{AZCB}$, if not it is a non-AZCB.

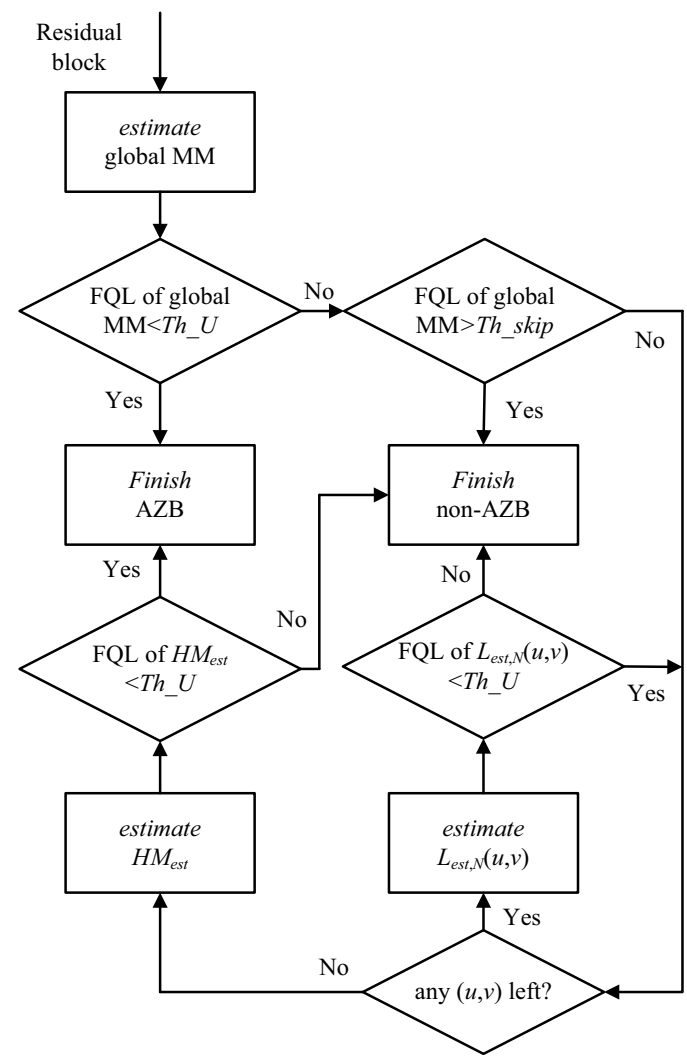

Figure 2. The flowchart of AZCB detection in UQ 


\section{Experimental results}

The following experimental results are based on HEVC test model version 13.0 (HM 13.0). The standard encoder_lowdelay_main.cfg configuration file is used. 21 typical test sequences are selected from Class A to Class F, with the quantization parameters (QP) set to 22, 27, 32, and 37 respectively. The change of BDPSNR, BDBR [8], and coding time are shown on Table 1. As Table 1 shows the average time saving of the proposed method is $50 \%$. The proposed method can greatly reduce coding efficiency with a negligible loss of PSNR and BD-rate.

Table 1. The Results for UQ AZCB Detection

\begin{tabular}{rrrr}
\hline Sequence & BDBR (\%) & BDPSNR (dB) & TS (\%) \\
\hline PeopleOnStreet & -0.04 & 0.002 & 44 \\
Traffic & -0.15 & 0.005 & 50 \\
BasketballDrive & 0.09 & 0.000 & 44 \\
BQTerrace & -0.07 & 0.001 & 51 \\
Cactus & -0.09 & 0.002 & 48 \\
Kimono & -0.07 & 0.002 & 42 \\
ParkScene & 0.03 & -0.001 & 53 \\
BasketballDrill & 0.09 & -0.004 & 49 \\
BQMall & -0.06 & 0.003 & 44 \\
PartyScene & -0.03 & 0.002 & 41 \\
RaceHorsesC & -0.05 & 0.002 & 36 \\
BasketballPass & 0.00 & -0.001 & 43 \\
BlowingBubbles & 0.14 & -0.005 & 41 \\
BQSquare & 0.13 & -0.005 & 50 \\
RaceHorsesD & 0.02 & -0.002 & 36 \\
FourPeople & 0.11 & -0.006 & 64 \\
Johnny & -0.04 & -0.001 & 69 \\
KristenAndSara & 0.12 & -0.003 & 65 \\
ChinaSpeed & -0.09 & 0.005 & 51 \\
SlideEditing & -0.07 & 0.010 & 69 \\
SlideShow & 0.10 & -0.007 & 63 \\
Average & 0.00 & 0.000 & 50 \\
\hline
\end{tabular}

The accuracy of AZCB detection process can be measured by false acceptance ration (FAR) and false rejection ratio (FRR). They can be calculated as equation (5),

$$
\begin{aligned}
& F R R=\frac{\text { num }_{m z}}{\text { num }_{z}} \\
& F A R=\frac{\text { num }_{m n}}{\text { num }_{n}}
\end{aligned}
$$

The num $_{m z}$ means the number of AZCB which are classified as non-AZCB incorrectly, and num $_{z}$ represents the real number of AZCB. While num $_{m n}$ and num $_{n}$ represent the number of non-AZCB which is misclassified as AZCB and the real number of nonAZCB. FRR means the ratio of AZCB which are classified as non-AZCB incorrectly, while FAR means the ratio of non-AZCB misclassified as AZCB. We want to detect AZCB as much as possible, which means that the FRR and FAR will be very low. In other words, higher detection efficiency means lower FRR and FAR. 
Table 2. Detection Efficiency in UQ by the Proposed Algorithm(FRR)

\begin{tabular}{rrrrr}
\hline Sequence & $\mathbf{4 \times 4}$ FRR $(\%)$ & $\mathbf{8 \times 8}$ FRR $(\%)$ & $\mathbf{1 6} \times \mathbf{1 6}$ FRR $(\mathbf{\%})$ & $\mathbf{3 2 \times 3 2}$ FRR $(\mathbf{\%})$ \\
\hline Class A & 1.2 & 3.2 & 5.2 & 13.7 \\
Class B & 1.8 & 3.5 & 5.5 & 12.2 \\
Class C & 2.8 & 6.2 & 9.2 & 19.0 \\
Class D & 2.1 & 7.1 & 10.4 & 20.5 \\
Class E & 1.0 & 2.4 & 3.4 & 7.2 \\
Class F & 1.1 & 3.0 & 4.4 & 7.0 \\
Average & 1.7 & 4.2 & 6.4 & 13.3 \\
\hline
\end{tabular}

The results of the proposed algorithm for UQ are shown as Table 2 and Table 3 . The average FRR of $4 \times 4,8 \times 8,16 \times 16$ and $32 \times 32$ TBs are $1.7 \%, 4.2 \%, 6.4 \%$ and $13.3 \%$ respectively. The average FRR of $4 \times 4,8 \times 8,16 \times 16$ and $32 \times 32$ TBs are $2.9 \%, 4.1 \%, 6.8 \%$ and $5.8 \%$ respectively.

Table 3. Detection Efficiency in UQ by the Proposed Algorithm(FAR)

\begin{tabular}{rrrrr}
\hline Sequence & $\mathbf{4 \times 4}$ FAR $(\%)$ & $\mathbf{8} \times \mathbf{8}$ FAR $(\mathbf{\%})$ & $\mathbf{1 6} \times \mathbf{1 6}$ FAR $(\mathbf{\%})$ & $\mathbf{3 2 \times 3 2}$ FAR $(\mathbf{\%})$ \\
\hline Class A & 3.0 & 4.3 & 6.5 & 4.0 \\
Class B & 3.0 & 4.4 & 7.0 & 6.3 \\
Class C & 2.4 & 3.4 & 5.0 & 3.4 \\
Class D & 3.2 & 4.4 & 6.1 & 5.0 \\
Class E & 3.2 & 5.2 & 10.5 & 10.8 \\
Class F & 2.3 & 3.1 & 6.2 & 5.7 \\
Average & 2.9 & 4.1 & 6.8 & 5.8 \\
\hline
\end{tabular}

Figure 3 compared the frame for both the proposed AZCB algorithm and the original HM test model. There are no notable differences between these images.
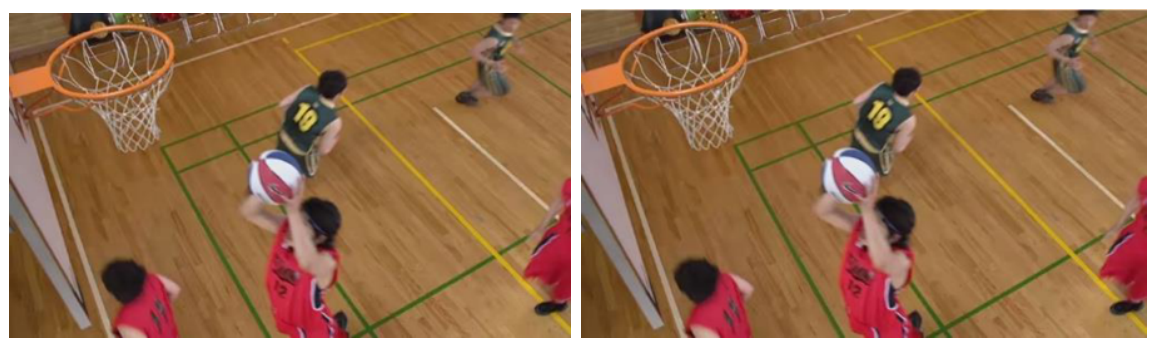

Figure 3. frame of proposed algorithm and HM

\section{Conclusion}

An AZCB detection algorithm for UQ is proposed in this paper. The algorithm adopts a low and high frequency coefficients separation strategy to estimate transform coefficients. Threshold to determine AZCB in UQ can be deduced directly. The proposed algorithms are implemented on HEVC test model to verify the efficiency. Experiment results show that the proposed algorithm can efficiently reduce the computation complexity while keeping nearly the same $\mathrm{RD}$ performance as with the original algorithm in HEVC. 


\section{References}

[1] International Telecommunication Union Telecommunication Standardization Sector (ITU-T) and International Organization for Standardization/International Electrotechnical Commission (ISO/IEC), "High efficiency video coding," Rec. H.265/ISO/IEC 23008-2, April 2013.

[2] Sullivan, G. J., Ohm, J., Han, W. J., \& Wiegand, T. Overview of the high efficiency video coding (hevc) standard. IEEE Transactions on Circuits \& Systems for Video Technology, 22(12), 2012, 1649-1668.

[3] Jung, S. W., Baek, S. J., Park, C. S., \& Ko, S. J. Fast mode decision using all-zero block detection for fidelity and spatial scalable video coding. IEEE Transactions on Circuits \& Systems for Video Technology, 20(2), 2010, 201-206.

[4] Ou, J. H. P. L. S. (2003). Motion search method based on all zero block detection in h.264. Signal Processing.

[5] Weicai Z, Jing L, Licheng J , et al. A novel early prediction method of all-zero coefficients in h.263. journal of electronics\&information technology, 2003, 25(4):573-576.

[6] Wang, H., \& Kwong, S. Hybrid model to detect zero quantized det coefficients in h.264. Multimedia IEEE Transactions on, 9(4), 2007, 728 - 735.

[7] Xie, Z., Liu, Y., Liu, J., \& Yang, T. A general method for detecting all-zero blocks prior to det and quantization. IEEE Transactions on Circuits \& Systems for Video Technology, 17(2),2007, 237-241.

[8] Bjontegaard, G. Calculation of average psnr difference between rd-curves. ITU-T VCEG-M33, April, 2001. 